\title{
The relationship between the prestellar core mass function and the stellar initial mass function
}

\author{
S. P. Goodwin ${ }^{1}$, D. Nutter ${ }^{2}$, P. Kroupa ${ }^{3}$, D. Ward-Thompson ${ }^{2}$, and A. P. Whitworth ${ }^{2}$ \\ 1 Department of Physics \& Astronomy, University of Sheffield, Hicks Building, Hounsfield Road, Sheffield, S3 7RH, UK \\ e-mail: S.Goodwin@sheffield.ac.uk \\ 2 School of Physics \& Astronomy, Cardiff University, Queens Buildings, The Parade, Cardiff CF24 3AA, UK \\ 3 Argelander-Institut für Astronomie, Universität Bonn, Auf dem Hügel 71, 53121 Bonn, Germany
}

Received 9 August 2007 / Accepted 2 November 2007

ABSTRACT

\begin{abstract}
Stars form from dense molecular cores, and the mass function of these cores (the CMF) is often found to be similar to the form of the stellar initial mass function (IMF). This suggests that the form of the IMF is the result of the form of the CMF. However, most stars are thought to form in binary and multiple systems, therefore the relationship between the IMF and the CMF cannot be trivial. We test two star formation scenarios - one in which all stars form as binary or triple systems, and one in which low-mass stars form in a predominantly single mode. We show that from a log-normal CMF, similar to those observed, and expected on theoretical grounds, the model in which all stars form as multiples gives a better fit to the IMF.
\end{abstract}

Key words. stars: formation - ISM: clouds - binaries: general

\section{Introduction}

The origin of the stellar initial mass function (IMF) is one of the outstanding unsolved problems in astrophysics. As stars form in dense molecular cores (see e.g. Ward-Thompson et al. 1994; Kirk et al. 2005; Ward-Thompson et al. 2007), it might well be expected that the IMF is related to the mass function of those cores (the CMF). This idea is supported by observations of prestellar cores, which show that their mass functions are often similar to the IMF of Galactic field stars (Motte et al. 1998; Testi \& Sargent 1998; Johnstone et al. 2000, 2001; Motte et al. 2001; Johnstone \& Bally 2006; Alves et al. 2007; Young et al. 2006; Nutter \& Ward-Thompson 2007; Simpson et al. 2007). Further support is given by the observation that Taurus may have both an unusual CMF (Onishi et al. 2002) and an unusual IMF (Luhman 2004; see also Goodwin et al. 2004c), although Kroupa et al. (2003) show that the IMF in Taurus may be compatible with the field IMF.

However, the relationship between the CMF and the IMF cannot be simple, as many, if not the vast majority, of stars form in binaries or higher-order multiple systems (see Goodwin \& Kroupa 2005; Duchêne et al. 2007; Goodwin et al. 2007, and references therein; see also Clark et al. 2007). Observations suggest that the binary frequency amongst young stars is higher than in the field (see Goodwin et al. 2007, and references therein) implying that binaries are destroyed by dynamical interactions in clusters (see Kroupa 1995a,b). However, Lada (2006) has argued that most M-dwarfs form as single stars, since the field M-dwarf binary fraction is relatively low and there is no need to invoke dynamical destruction of low-mass binaries to form these (single) stars. The opposing view is argued by Goodwin \& Kroupa (2005) and Goodwin \& Whitworth (2007).

If stars (or at least relatively high-mass stars) usually form in small- $N$ multiples then there cannot be a trivial one-to-one relationship between the IMF to the CMF. Firstly, the mass of a core is distributed between a number of stars. Secondly, some stars are expected to be ejected at an early age from small- $N$ multiples (e.g. Reipurth \& Clarke 2001; Goodwin et al. 2007, and references therein; see also Sect. 3). Thirdly, many binary systems are expected to be destroyed in clusters (Kroupa 1995a,b; Kroupa et al. 2003; Goodwin \& Whitworth 2007; also see Goodwin et al. 2007, and references therein). Thus the CMF should relate most closely to the initial system mass function which, in turn, is modified by dynamical effects to produce a mixture of single and multiple systems.

In this paper we examine the relationship between the IMF and the CMF, in particular we use the new results for the CMF in Orion from Nutter \& Ward-Thompson (2007). In Sect. 2 we review observations of the CMF, in Sect. 3 we present our general method, and in Sect. 4 we compare the IMFs we produce with the observations.

\section{Observations of the CMF}

The first observational link between the IMF and the CMF was made by Motte et al. (1998) in a millimetre study of the $\rho$-Ophiuchi molecular cloud. They found that the high-mass slope of the CMF matched that of the IMF. This result has been confirmed for Ophiuchus (Johnstone et al. 2000; Young et al. 2006; Simpson et al. 2007) and a number of other nearby clouds, including Orion (Motte et al. 2001; Johnstone et al. 2001; Johnstone \& Bally 2006; Nutter \& Ward-Thompson 2007), the Pipe Nebula (Alves et al. 2007), and Taurus (Onishi et al. 2002; however see Goodwin et al. 2004c), as well as for more distant massive star-forming regions such as NGC 7538 and M 17 (Reid \& Wilson 2006a,b).

While the slope of the CMF seems to be consistent from region to region, the position of the peak of the CMF appears to shift from $\sim 0.1 M_{\odot}$ in nearby low-mass regions such as $\rho$-Ophiuchus (e.g. Motte et al. 1998), to a higher mass of 
$\sim 1 M_{\odot}$ in more distant and massive star-forming regions such as Orion (e.g. Nutter \& Ward-Thompson 2007). Very massive starforming regions such as $\mathrm{M} 17$ show a flattening of the CMF at an even higher mass of $\sim 8 M_{\odot}$ (Reid \& Wilson 2006a,b), though the data are incomplete before a turn-over is seen. Whether this is an intrinsic effect where the mass of the peak in the CMF is related to the mass of the stars being formed, or an observational effect caused by the blending of multiple sources at larger distances, is not yet known.

\section{The relationship between core and stellar mass functions}

We assume that the star formation properties of a core may be described by three basic parameters: the mass of the core $M_{\mathrm{C}}$, the efficiency with which the core turns gas into $\operatorname{star}(\mathrm{s}) \epsilon$ (so that the total mass of stars is $\epsilon M_{\mathrm{C}}$ ), and the number of stars formed within a core $N_{*}$ (the choices of $N_{*}$ are discussed in Sect. 4). We note that $\epsilon$ and $N_{*}$ may well be functions of $M_{\mathrm{C}}$.

There are then two basic distribution functions of the stars. The multiple system mass function (MSMF) is the mass distribution of the multiple systems produced by cores. The single star mass function (SSMF) is the mass distribution of all of the individual stars formed in all of the single and multiple systems in all of the cores. It is the SSMF that will correspond to the (binary corrected) initial mass function (IMF; see below).

Note that the MSMF will evolve due to several effects. Firstly, unstable high-order multiple systems may decay, preferentially ejecting single, low-mass stars (e.g. Reipurth \& Clarke 2001; Bate et al. 2002, 2003; Sterzik \& Durisen 2003; Goodwin et al. 2004a,b; Delgado Donate et al. 2004a,b; Hubber \& Whitworth 2005; Umbriet et al. 2005). Secondly, binaries may be "destroyed" by rather violent close binary-binary/binarysingle interactions (Kroupa 1995a,b), and thirdly, wide, lowmass binaries may be "disrupted" by more gentle impulses from passing stars (Goodwin \& Whitworth 2007).

\subsection{From a CMF to an IMF}

The procedure for generating an IMF (via a MSMF and SSMF) from a CMF is very simple. We randomly sample a core mass from a CMF (see Sect. 3.3 for our choice of CMF). This core then produces $N_{*}$ stars of total mass $\epsilon M_{\mathrm{C}}$ (this is similar to the approach of Sterzik et al. 2001, however we do not constrain the IMF of the stars in any way except through the CMF of the cores).

The masses of the $N_{*}$ components in a multiple system are chosen randomly. In a binary system $\left(N_{*}=2\right)$, the masses of the primary and secondary are selected from a flat mass ratio distribution (i.e. one random number U[0,1]). In a higher-order multiple the masses are distributed randomly, i.e. $N_{*}$ random numbers $U[0,1]$ are chosen and then the sum is normalised to unity to provide the mass distribution.

The SFE, $\epsilon$, is chosen to provide the best fit to the canonical IMF (see below) and is assumed to be constant for all cores. It might be thought that SFE should depend on the mass of stars formed, as feedback energy increases with increasing stellar mass. However, the potential well from which gas must be removed by feedback also increases with increasing stellar and gas mass and so possibly the SFE is constant, or even increasing with mass. Given the uncertainties involved we make the simplest assumption possible that the SFE is constant. As will be seen, a good fit to the IMF can be obtained while making this

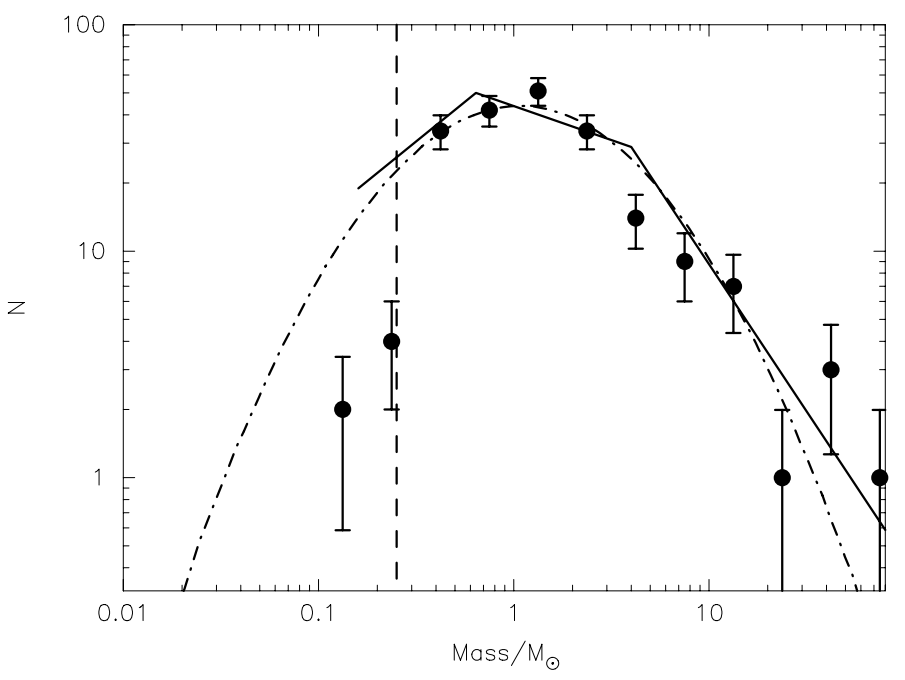

Fig. 1. The CMF of Nutter \& Ward-Thompson (2007) with $\sqrt{N}$ errorbars, the completeness limit of the observations is marked by the vertical dashed line. The data are fitted by a log-normal distribution with mean $\mu_{\log _{10} M}=0.05$ and $\sigma_{\log _{10} M}=0.55$ (dashed-dot line). Also plotted for comparison is the canonical IMF (Eq. (1)) with the same slopes $\left(\alpha_{1}=0.3, \alpha_{1}=1.3\right.$ and $\left.\alpha_{1}=2.3\right)$, but turn-over masses increased by a factor of $8\left(M_{0}=0.16 M_{\odot}, M_{1}=0.64 M_{\odot}, M_{2}=4 M_{\odot}\right.$ and $\left.M_{3}=80 M_{\odot}\right)$.

assumption and there appears no need to appeal to a variation of SFE between cores of different masses.

\subsection{The canonical observed IMF}

We assume that the actual underlying IMF of stars has the canonical form (Kroupa 2002; see also Kroupa 2007)

$N(M) \propto \begin{cases}M^{-\alpha_{1}} & M_{0} \leq M \leq M_{1} \\ \left(\frac{M_{1}^{-\alpha_{1}}}{M_{1}^{-\alpha_{2}}}\right) M^{-\alpha_{2}} & M_{1} \leq M \leq M_{2} \\ \left(\frac{M_{1}^{-\alpha_{1}}}{M_{1}^{-\alpha_{2}}}\right)\left(\frac{M_{2}^{-\alpha_{2}}}{M_{2}^{-\alpha_{3}}}\right) M^{-\alpha_{3}} & M_{2} \leq M \leq M_{3}\end{cases}$

with $\alpha_{1}=0.3, \alpha_{2}=1.3$ and $\alpha_{3}=2.3$ as the slopes, and $M_{0}=$ $0.02 M_{\odot}, M_{1}=0.08 M_{\odot}, M_{2}=0.5 M_{\odot}$ and $M_{3}=10 M_{\odot}$ as the masses of the limits and turning points of the IMF. This form of the IMF matches well other recent determinations of the IMF (e.g. Chabrier 2003).

The canonical IMF is corrected for the presence of unresolved binary systems and therefore the IMF should follow the SSMF.

\subsection{Forms of the CMF}

Our standard CMF is that determined for Orion by Nutter \& Ward-Thompson (2007). We model this as a log-normal with dispersion $\sigma_{\log _{10} M}=0.55$ and a mean of $\mu_{\log _{10} M}=0.05$ as illustrated in Fig. 1. This CMF is not too dissimilar to an IMF-like distribution with the slopes $\alpha$ remaining the same, but turn-over masses of $\sim 8$ times those in the canonical IMF (Eq. (1)). We also note that this is very similar to the CMF of the Pipe dark cloud (Alves et al. 2007).

Our fit to the Nutter \& Ward-Thompson (2007) CMF is above the final two points which are below the completeness limit. We assume that the entire core mass distribution is modelled by a log-normal which would be expected on theoretical 
grounds (Padoan \& Nordlund 2002, 2004; Klessen \& Burkert 2000; Klessen 2001; Li et al. 2003; Jappsen et al. 2005) even in a wide variety of physical conditions (see esp. Padoan \& Nordlund 2002; Jappsen et al. 2005). We also note that Padoan \& Nordlund (2002, 2004) tend to find a very steep decline in the CMF below the peak which would be compatible with these observations.

Clark et al. (2007) recently noted that the free-fall times of clumps of different masses are different and that low-mass cores collapse significantly faster than higher-mass cores. Thus an observed CMF must be constantly replenished with low-mass cores in order to retain a constant form. We assume that our CMF represents a "snapshot" of the CMF. If star formation occurs rapidly in clusters (in <1 Myr, e.g. Elmegreen 2000) then the observed CMF should represent the total CMF for masses above the peak of the $\mathrm{CMF}$ as the free-fall time for cores $>1 M_{\odot}$ is a significant fraction of the cluster formation timescale (Clark et al. 2007, see their Fig. 1).

\section{Results}

\subsection{The fully multiple model}

Goodwin \& Kroupa (2005) suggested that the observed properties of multiple systems could be reproduced if each core produces 2 or 3 stars. Single field stars are then produced by the dynamical decay and destruction of multiple systems in young clusters (Kroupa 1995a,b; Goodwin \& Kroupa 2005; Goodwin \& Whitworth 2007; Goodwin et al. 2007, and references therein).

To model the fully multiple scenario we assume that cores of mass $\epsilon M_{\mathrm{C}}<0.75 M_{\odot}$ form entirely binary systems, and cores with $\epsilon M_{\mathrm{C}} \geq 0.75 M_{\odot}$ form multiple systems with a ratio of 3:1 binaries-to-triples. The SFE is chosen to give a good fit to the canonical IMF with $\epsilon=0.27$.

In this scenario the initial multiplicity fraction is unity. Single stars and brown dwarfs are produced by the destruction of many (especially low-mass) initially multiple systems (see Sect. 3).

The result of the fully multiple model are illustrated in Fig. 2. This model produces a good fit to the canonical IMF for all masses except the very highest. The mass functions dip below the canonical slope of -1.3 at high masses due to the steep decline of the log-normal CMF at high masses ${ }^{1}$.

The fully multiple model requires the dynamical destruction (see e.g. Kroupa 1995b; Kroupa et al. 2003; Goodwin \& Kroupa 2005; Goodwin et al. 2007) of significant numbers of low-mass binary systems in young clusters in order to change the initial binary fraction of unity to the field value.

We note that in this model brown dwarfs are not primarily produced as single objects in cores ("star-like" formation, e.g. Padoan \& Nordlund 2004), nor as ejected embryos from high-mass cores (the ejection hypothesis, e.g. Reipurth \& Clarke 2001). Instead they mainly form as the distant companions to M-dwarfs which are then disrupted. This is the scenario proposed by Goodwin \& Whitworth (2007) as a major mode of brown dwarf formation. We note that this might be consistent with the idea that brown dwarfs form as a separate population of objects, possibly with a discontinuous IMF (Kroupa et al. 2003; Thies \& Kroupa 2007; Kumar \& Schmeja 2007).

${ }^{1}$ It might be expected that the actual CMF continues as a power-law decline rather than being fitted by a log-normal at high masses (see e.g. Padoan \& Nordlund 2002, 2004).

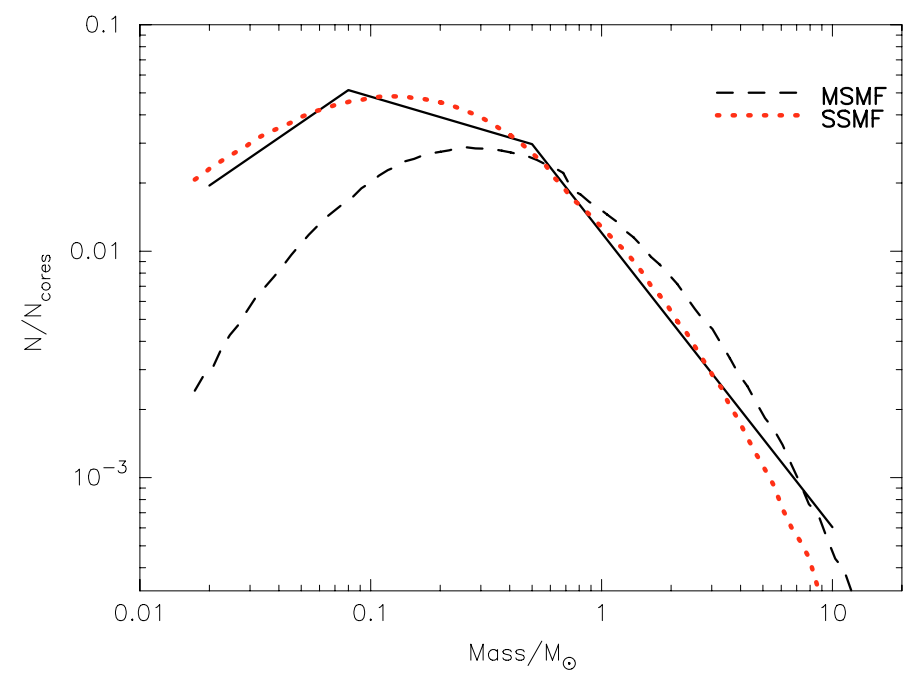

Fig. 2. The fully multiple model with $N_{\star}=2$ for systems with $\epsilon M_{\mathrm{C}}<$ $0.75 M_{\odot}$ and $N_{\star}=3: 1$ binary-to-triple ratio for $\epsilon M_{\mathrm{C}} \geq 0.75 M_{\odot}$, and $\epsilon=0.27$. The initial MSMF (dashed line), and the SSMF (red dotted line) compared to the canonical IMF (thin solid lines).

\subsection{The low-mass single star model}

Lada (2006) suggests that M-dwarfs tend to form as single stars, since most M-dwarfs in the field (roughly $55 \%$ by total number) are single stars. In this picture, destructive dynamical processes are unimportant for low-mass stars. However, Solar-type stars (and higher-masses) must still usually form in multiple systems to fit the observed high multiplicity fraction of T Tauri stars (e.g. Mathieu 1994; Patience et al. 2002; Duchêne et al. 2007; Goodwin et al. 2007, and references therein).

We model the situation where most low-mass stars form as singles (the low-mass single star model) by making $N_{*}$ a strong function of the core mass. Cores with $\epsilon M_{\mathrm{C}}<0.5 M_{\odot}$ form stars in a 2:1 single-to-binary ratio. Cores with masses $0.5<\epsilon M_{\mathrm{C}} / M_{\odot}<1 M_{\odot}$ have a $1: 1$ ratio of singles-to-binaries. And cores with $\epsilon M_{\mathrm{C}} \geq 1 M_{\odot}$ have a 3:1 binary-to-triple ratio (as in the fully multiple model). These probabilities roughly reflect a combination of the low-mass field (M-dwarf, see Fischer \& Marcey 1992; also Lada 2006) and intermediate-mass PMS binary fractions (see Duchêne et al. 2007; Goodwin et al. 2007, and references therein).

The results from this model are shown in Fig. 3 with a SFE of $\epsilon=0.15$ which provides the best (but still not a good) fit to the canonical IMF. This model cannot be made to fit the canonical IMF well with any choice of $\epsilon$.

The main problem with the low-mass single star model is that it provides no mechanism for forming brown dwarfs other than as single brown dwarfs in very low mass cores. As the CMF peaks at $\sim 1 M_{\odot}$ there are very few cores below $0.1 M_{\odot}$ and so the SFE must be made very low in order to shift the peak of the CMF to the peak of the IMF. This is avoided in the fully multiple model by producing brown dwarfs as companions to low-mass stars. Increasing the SFE to greater than $15 \%$ results in far too few VLMOs and also shifts the peak of the IMF to too-high a mass. We also note that a SFE of $15 \%$ suggests that a significant number of cores produce planetary-mass objects (which are not shown in Fig. 3).

There are two ways we might possibly escape from the problem of too-few brown dwarfs. Firstly, we may postulate a very large population of low-mass cores from which brown dwarfs can form (e.g. Padoan \& Nordlund 2004). In this situation the 




Fig. 3. Low-mass single star model with $N_{\star}=2: 1$ single:binary for $\epsilon M_{\mathrm{C}}<0.5 M_{\odot}, 1: 1$ single:binary for $0.5<\epsilon M_{\mathrm{C}} / M_{\odot}<1$, and $3: 1$ binary:triple for $\epsilon M_{\mathrm{C}} \geq 1 M_{\odot}$, with $\epsilon=0.15$. The initial MSMF (dashed line), and the SSMF (red dotted line) compared to the canonical IMF (thin solid lines).

vast majority of cores would be below the peak of the CMF. However, the simulations of Padoan \& Nordlund (2002, 2004) suggest that the CMF should drop sharply below the peak, far faster than a log-normal distribution. Thus, there appears to be no good reason for expecting a large population of low-mass cores (see Goodwin \& Whitworth 2007, for a number of other reasons why significant numbers of low-mass brown dwarfforming cores would not be expected).

Secondly, we may form brown dwarfs by ejecting low-mass embryos from massive cores, thus creating brown dwarfs (e.g. Reipurth \& Clark 2001). However, large numbers of ejections per high-mass core would be required to create almost all of the brown dwarf population in this way, and large numbers of such ejections have consequences that are difficult to reconcile with observations (see e.g. Goodwin \& Kroupa 2005; Goodwin et al. 2007; Whitworth et al. 2007, and references therein; Goodwin \& Whitworth 2007).

The slope of the high-mass end of the IMF is also far too steep $(\sim 1.6$ rather than the observed $\sim 1.3)$. This is due to the change in the modes of fragmentation at $\epsilon M_{\mathrm{C}}=0.5 M_{\odot}$ and $1 M_{\odot}{ }^{2}$. This is because at high masses the mass of stars is divided between 2 or 3 stars, while at lower masses it is divided only between 1 or 2 . Thus altering the modes of fragmentation changes the slope from the IMF-like slope of the CMF to a steeper slope. The upper-mass slope only matches the slope of the CMF if fragmentation is independent of mass for cores above the knee in the IMF at $0.5 M_{\odot}$.

The problem with the upper-mass slope can be alleviated somewhat by assuming that all stars form with the field binary fraction (rising from $33 \%$ for M-dwarfs to $60 \%$ for G-dwarfs). However, this solution conflicts with observations that the initial binary fraction for stars $>1 M_{\odot}$ is consistent with unity (Goodwin \& Kroupa 2005; Duchêne et al. 2007; Goodwin et al. 2007). These observations suggest that there must be a fairly rapid transition between 0.5 and $1 M_{\odot}$ from a low to a high

\footnotetext{
${ }^{2}$ Small jumps may be seen at $0.5 M_{\odot}$ and $1 M_{\odot}$ as the function steps suddenly between different modes rather than being smooth, however a smooth function merely evens-out these jumps but does not change the overall appearance of the IMF.
}

primordial binary fraction which will result in too-steep an upper-mass slope of the IMF.

We have assumed that the mass ratio of binaries is a flat distribution. Biasing the mass ratio distribution to low- $q$ (i.e. highly unequal mass systems) improves the problems at the high-mass end of the IMF slightly. If most high-mass cores produce one large star and one or two very low-mass stars, then the IMF at the high-mass end becomes more similar to the MSMF (as this is dominated by one of the stars). However, the mass ratio distribution needs to be very biased for this to have a significant effect.

The too-steep slope of the upper-end of the IMF can also be solved by assuming that the SFE increases with increasing core mass (in just the right way). However, we feel this solution is unlikely as the SFE would have to be fine-tuned to give the correct slope and it would seem peculiar to postulate that lowmass cores produce stars at very low efficiencies $(\sim 10 \%)$, whilst higher-mass cores are able to convert more of their gas $(\sim 30 \%)$ into stars (the opposite of what might be expected from arguments based on feedback).

\section{Discussion}

We have shown that the observed mass functions of cores in Orion B (Nutter \& Ward-Thompson 2007) can give rise to the IMF of stars. In particular we have shown that, to produce the stellar and sub-stellar IMF, the majority of these cores must fragment into multiple systems. However, there are a number of issues about cores and the CMF that are worth discussing in this context.

It should be noted that it may not be fragmentation into "cores" in clusters that sets the IMF of stars. If competitive accretion (see Bonnell et al. 2007, and references therein) is the dominant process, then the CMF at best acts to set the initial masses upon which competitive accretion begins to work. In such a scenario there would be little or no relationship between the $\mathrm{CMF}$ and the IMF.

However, we would argue that the form of the CMF in diffuse star forming regions does have a direct relevance to the origin and form of the IMF. Given the apparent universality of the IMF across a wide range of star forming environments (e.g. Kroupa 2002) we are presented with two options. Firstly, that the mechanism(s) that produce the IMF are fundamentally different in different environments, but they always produce the same outcome. Or, secondly, that there is a single, underlying, mechanism that produces the IMF in all environments. The latter possibility appeals due to its simplicity, and would suggest that the form of the CMF is the driving factor in establishing the form of the IMF, and that the form of the $\mathrm{CMF}$ is roughly the same in diffuse and clustered regions (even if the cores themselves are different in spacial size). Indeed, simulations of turbulence always seem to produce roughly log-normal CMFs whatever the environment.

\section{Conclusions}

We have examined the relationship between the core mass function (CMF) and the stellar initial mass function (IMF). We use the Orion CMF from Nutter \& Ward-Thompson (2007) as a "standard" which we fit using a log-normal distribution. We note that this CMF is not dissimilar to the stellar (Kroupa 2002) IMF shifted upwards in mass by a factor of 8 (see also Alves 2007). We randomly sample cores from the CMF and assumed that each core produces a certain number of stars with a random distribution of masses between the components. 
The canonical IMF is reproduced very well by a scenario in which every low-mass cores fragment into binaries, and highmass cores fragment into a multiple system with a ratio of binaries-to-triples of 3:1 (see e.g. Goodwin \& Kroupa 2005) and a star formation efficiency (SFE) of $\sim 30 \%$. Dynamical disruption (Kroupa 1995a,b; Goodwin \& Whitworth 2007) of systems then evolves the initial binary fraction of unity into the field population.

We find that a scenario in which low-mass stars preferentially form single systems (e.g. Lada 2006) cannot reproduce the observed IMF from a log-normal CMF. Firstly, the slope of the high-mass IMF is too steep. Secondly, and most seriously, this model cannot reproduce the correct numbers of brown dwarfs to high-mass stars. The best-fit to the canonical IMF is found when the SFE is only $\sim 15 \%$. Such a low SFE is required, as the only way in which brown dwarfs may be produced in significant numbers is through the formation of a single brown dwarf from a core. Higher SFEs are required to produce sufficient high-mass stars, however such SFEs significantly underproduce brown dwarfs and low-mass stars.

A lingering question is the value of the star formation efficiency that must be applied to fit the IMF. The best-fit value of $\epsilon$ in the fully multiple model suggest that only $\sim 30 \%$ of the mass in a core ends-up in the stars which that core forms (a similar value for the SFE is found by Alves et al. 2007). This seems a very low value and may suggest that the determinations of the absolute core masses are wrong. Another possibility is that feedback from jets is far more efficient than previously thought and manages to disperse most of the gas initially in the core. A final possibility is that we are not observing "typical" cores which produce the IMF and that the observed CMFs will produce somewhat top-heavy IMFs (cf. Taurus, Goodwin et al. 2004c).

We conclude that a model in which all stars and brown dwarfs form in multiple systems from a log-normal core mass distribution provides a very good fit the observed IMF.

Acknowledgements. S.P.G. was supported during some of this work by a UKAFF Fellowship. S.P.G. also acknowledges the support and hospitality of the International Space Science Institute in Bern, Switzerland where part of this work was done as part of a International Team Programme. S.P.G. and P.K. also acknowledge support from a Royal Society International Joint Project grant. P.K. also thanks the Leverhulme Trust for the award of a Leverhulme Trust Visiting Professorship at Sheffield University.

\section{References}

Alves, J., Lombardi, M., \& Lada, C. J. 2007, A\&A, 462, 17

Bate, M. R., Bonnell, I. A., \& Bromm, V. 2002, MNRAS, 332, L65

Bate, M. R., Bonnell, I. A., \& Bromm, V. 2003, MNRAS, 339, 577

Bonnell, I. A., Larson, R. B., \& Zinnecker, H. 2007, in Protostars and Planets V, ed. B. Reipurth, D. Jewitt, \& K. Keil (Tuscon: University of Arizona Press), 149

Briceño, C., Luhman, K. L., Hartmann, L., Stauffer, J. R., \& Kirkpatrick, J. D. 2002, ApJ, 580, 317

Chabrier, G. 2003, PASP, 115, 763

Clark, P. C., Klessen, R. S., \& Bonnell, I. A. 2007, MNRAS, 379, 57
Delgado Donate, E. J., Clarke, C. J., Bate, M. R., \& Hodgkin, S. T. 2004a, MNRAS, 351, 617

Delgado Donate, E. J., Clarke, C. J., \& Bate, M. R. 2004b, MNRAS, 347, 759

Duchêne, G., Delgado Donate, E., Haisch, K. E., Jr, Loinard, L., \& Rodriguez, L. F. 2007, in Protostars and Planets V, ed. B. Reipurth, D. Jewitt, \& K. Keil (Tuscon: University of Arizona Press), 379

Elmegreen, B. G. 2000, ApJ, 530, 277

Fischer, D. A., \& Marcy, G. W. 1992, ApJ, 396, 178

Goodwin, S. P., \& Kroupa, P. 2005, A\&A, 439, 565

Goodwin, S. P., \& Whitworth, A. P. 2007, A\&A, 466, 943

Goodwin, S. P., Whitworth, A. P., \& Ward-Thompson, D. 2004a, A\&A, 414, 633

Goodwin, S. P., Whitworth, A. P., \& Ward-Thompson, D. 2004b, A\&A, 423, 169

Goodwin, S. P., Whitworth, A. P., \& Ward-Thompson, D. 2004c, A\&A, 419, 543

Goodwin, S. P., Whitworth, A. P., \& Ward-Thompson, D. 2006, A\&A, 452, 487

Goodwin, S. P., Kroupa, P., Goodman, A., \& Burkert, A. 2007, in Protostars and Planets V, ed. B. Reipurth, D. Jewitt, \& K. Keil (Tuscon: University of Arizona Press), 133

Hubber, D. A., \& Whitworth, A. P. 2005, A\&A, 437, 113

Jappsen, A.-K., Klessen, R. S., Larson, R. B., Li, Y., \& Mac Low, M.-M. 2005, A\&A, 435, 611

Johnstone, D., \& Bally, J. 2006, ApJ, 653, 383

Johnstone, D., Wilson, C. D., Moriarty-Schieven, G., et al. 2000, ApJ, 545, 327

Johnstone, D., Fich, M., Mitchell, G. F., \& Moriarty-Schieven, G. 2001, ApJ, 559,307

Kirk, J. M., Ward-Thompson, D., \& André, P. 2005, MNRAS, 360, 1506

Klessen, R. S. 2001, ApJ, 556, 837

Klessen, R. S., \& Burkert, A. 2000, ApJS, 128, 287

Kroupa, P. 1995a, MNRAS, 277, 1491

Kroupa, P. 1995b, MNRAS, 277, 1507

Kroupa, P. 2002, Science, 295, 82

Kroupa, P. 2007 [arXiv:astro-ph/0703124]

Kroupa, P., Bouvier, J., Duchêne, G., \& Moraux, E. 2003, MNRAS, 346, 354

Kumar, M. S. N., \& Schmeja, S. 2007, A\&A, 471, 33

Lada, C. J. 2006, ApJ, 640, L63

Lada, C. J., \& Lada, E. A. 2003, ARA\&A, 41, 57

Li, Y., Klessen, R. S., \& Mac Low, M.-M. 2003, ApJ, 592, 975

Luhman, K. L. 2004, ApJ, 617, 121

Mathieu, R. D. 1994, ARA\&A, 32, 465

Maxted, P. F. L., \& Jeffries, R. D. 2005, MNRAS, 362, L45

Motte, F., André, P., \& Neri, R. 1998, A\&A, 336, 150

Motte, F., André, P., Ward-Thompson, D., \& Bontemps, S. 2001, A\&A, 372, L41

Muench, A. A., Lada, E. A., Lada, C. J., \& Alves, J. 2002, ApJ, 573, 366

Nutter, D., \& Ward-Thompson, D. 2007, MNRAS, 374, 1413

Onishi, T., Mizuno, A., Kawamura, A., Tachihara, K., \& Fukui, Y. 2002, ApJ, 575,950

Padoan, P., \& Nordlund, § 2002, ApJ, 576, 870

Padoan, P., \& Nordlund, А 2004, ApJ, 617, 559

Patience, J., Ghez, A. M., Reid, I. N., \& Matthews, K. 2002, AJ, 123, 1570

Pinfield, D. J., Dobbie, P. D., Jameson, R. F., et al. 2003, MNRAS, 342, 1241

Reid, M. A., \& Wilson, C. D. 2006a, ApJ, 644, 990

Reid, M. A., \& Wilson, C. D. 2006b, ApJ, 650, 970

Reipurth, B., \& Clarke, C. J. 2001, AJ, 122, 432

Simpson, R., Nutter, D., \& Ward-Thompson, D. 2007, in prep.

Sterzik, M. F., \& Durisen, R. H. 2003, A\&A, 400, 1031

Sterzik, M. F., Durisen, R. H., \& Pickett, B. K. 2001, A\&A, 371, 952

Testi, L., \& Sargent, A. 1998, ApJ, 508, L91

Thies, I., \& Kroupa, P. 2007, ApJ, submitted

Umbreit, S., Burkert, A., Henning, T., Mikkola, S., \& Spurzem, R. 2005, ApJ, 623,940

Ward-Thompson, D., Scott, P. F., Hills, R. E., \& André, P. 1994, MNRAS, 268, 276

Ward-Thompson, D., Andre, P., Crutcher, R., et al. 2007, in Protostars and Planets V, ed. B. Reipurth, D. Jewitt, \& K. Keil (Tuscon: University of Arizona Press), 33

Whitworth, A. P., Bate, M. R., Nordlund, Å., Reipurth, B., \& Zinnecker, H. 2007, in Protostars and Planets V, ed. B. Reipurth, D. Jewitt, \& K. Keil (Tuscon: University of Arizona Press), 459

Young, K. E., Enoch, M. L., Evans, N. J., II, et al. 2006, ApJ, 644, 326 